\title{
Projeto digital de edifícios em altura por meio de tesselação e mapeamento
}

\author{
Digital Design of High-Rise Buildings with Tessellation and Mapping \\ - Lilian Maciel Furtado Silva \\ UniCEUB, Brazil \\ Lilianmfs91@gmail.com \\ - Neander Furtado Silva \\ UnB, Brazil \\ Neander.furtado@gmail.com
}

\begin{abstract}
A new high-rise building, when inserted in an existing urban environment, cannot have all the floor plans identical since the visual and environmental constraints change from floor to floor due to the interference of neighboring buildings, sun light, prevailing wind and noise. So how can we design a building that takes into consideration all this variables? How can digital design tools help? (DELPINO, 2014).

We believe that a building like that can be designed constructing facade maps which allow a floor by floor analysis considering visual, wind, noise and sun light constraints. We believe that facade maps analysis and their superposition provide clues of how each floor plan can be built to provide comfort and to take most advantage of the view. (DELPINO, 2014).

We believe that variation techniques such as tessellation and morphing can provide the formal bases for such design. We believe that digital design tools are vital both for analysis as well as for designing this type of building. (IWAMOTO, 2009, p. 35-59; MOUSSAVI, 2009, p. 42-216).
\end{abstract}

Keywords: High-rise Buildings, Digital Design Method, Tessellation, Mapping

\section{Introdução}

$\mathrm{O}(\mathrm{a})$ arquiteto(a) em geral se depara com dificuldades de caráter contextual quando projeta um novo edifício em altura para um ambiente urbano já existente. Muitas vezes precisa escolher que condicionantes relacionadas ao entorno irá atender e quais irá deixar em segundo plano. Por exemplo, pode haver dificuldades em usufruir de uma vista agradável e evitar, ao mesmo tempo, intensa incidência solar. Por isto, esta pesquisa propõe uma solução diferenciada para cada andar de forma a, por exemplo, atender condicionantes ambientais e também usufruir de vistas desejáveis.

\section{Problema de Pesquisa}

A projetação ideal de um novo edifício em altura, quando inserido em um contexto urbano existente, não pode ter todos andares idênticos, uma vez que as vistas e condicionantes ambientais mudam de andar por andar, devido às interferências dos edifícios vizinhos e aos ruídos provenientes do trânsito, dos pedestres, etc. (Delpino, 2014)

\section{Hipótese}

Nossa hipótese de trabalho neste projeto de pesquisa foi no sentido de que é possível projetar um edifício em altura que responda às análises dos condicionantes do terreno e do entorno por meio de mapeamento, andar por andar, considerando ventos predominantes, insolação, intensidade dos ruídos, permeabilidade visual, vistas desejáveis e indesejáveis. (Delpino, 2014)

A justificativa de nossa hipótese é devida ao fato de que estes mapeamentos de fachadas, quando são sobrepostos, se tornam instrumentos de análise importantes e até mesmo imprescindíveis para definir como cada pavimento do edifício em altura pode ser construído para obter conforto e vistas desejáveis. Nesta pesquisa esses mapeamentos foram gerados a partir de recursos digitais de modelagem e simulação.

A técnica de Tesselação que ocorre por meio decomposição e variação da forma geométrica (Iwamoto, 2009, p. 35-59; Moussavi, 2009, p. 42-216), que utilizamos nesta pesquisa, podem fornecer as bases formais, estruturais, de escolha de materiais e etc., para este tipo de projeto de edificação. 


\section{Método de Pesquisa}

Iniciamos nosso trabalho utilizando o conceito de Tesselação, que por definição parte do princípio de que a forma final a ser criada é construída por repetições de uma ou mais formas geométricas sobre um plano sem que haja espaços entre elas e nem sobreposições.

"Tesselação é a junção de partes que se encaixam sem lacunas para formar um plano ou superfície. Tesselações podem virtualmente ser de qualquer forma desde que as peças se unam em formação compacta. Os desenhos geometricamente padronizados de M. C. Escher são frequentemente citados como um exemplo de Tesselação. Em arquitetura este termo se refere a ambos painéis ou murais padronizados e digitalmente definidos como padrões de malha" (Iwamoto, 2009, p. 36.)

Apresentamos a seguir uma imagem de Tesselação(Figura 1.1) conhecida da obra de Escher que inspirou nosso método de trabalho, principalmente a escolha da forma da célula base utilizada na construção de nosso edifício em alturas.

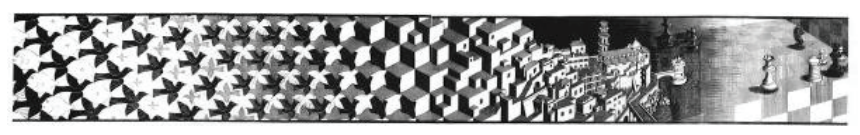

Figure 1.1: Metamorfoses de M.C. Escher Source: http://www. mcescher.com/gallery/transformation-prints/

A Tesselação vem sendo usada na confecção de mosaicos desde a Roma Antiga, Império Bizantino, nas paredes da Arquitetura Islâmica até os vitrais coloridos das catedrais góticas. Essas superfícies decorativas permitem filtrar a luz ou a vista, definem os espaços, transmitem significado simbólico.

Outro exemplo conhecido de Tesselação mais simples do que aqueles do Escher são os mosaicos da Roma Antiga como mostra a Figura 1.2.

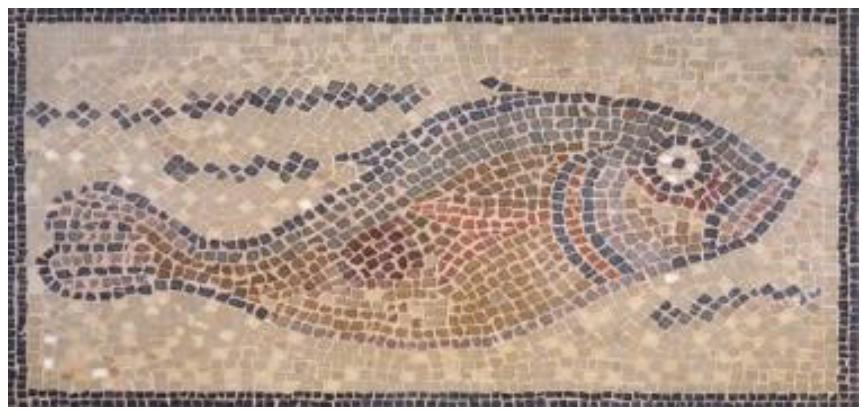

Figure 1.2: Mosaico de Peixe Source:www.okeanosgroup.com

Outro exemplo de Tesselação também muito conhecido são as formas da cobertura da Opera de Sydney (1973), projetada por Jorn Utzon. Essas formas foram esboçadas pela superfície de segmentos extraídos de esferas de raios variados e depois subdividos em porções planas (Figura 1.3).

Frank Gehry também utilizou triangulação, uma forma de Tesselação planar, na cobertura de vidro do DG Bank em Parizer Platz (Figura 1.4), Berlin. (Kolarevic, 2003, p. 45.).

Neste trabalho fizemos diversas simulações volumétricas de edifício em altura, a partir de uma malha hexagonal, inspirada na obra de metamorfoses de M. C. Escher. Este autor utilizou o conceito de Tesselação quando desenhou, repetidamente e sem sobreposição, um grupo de pássaros voando, os quais transformou, gradativamente, em formas geométricas e estas, por último, em uma pequena cidade antiga (Figura 1.1). Segundo nossa interpretação (Figura 1.5) esta obra do M.C. Escher, Metamorfoses III, poderia ter sido feita por meio de tesselações de hexágonos e por isto geramos uma malha hexagonal para projetar o nosso edifício. Geramos volumes hexagonais que seguem essa malha hexagonal para simular as combinações da composição desses volumes (Figura 1.6).

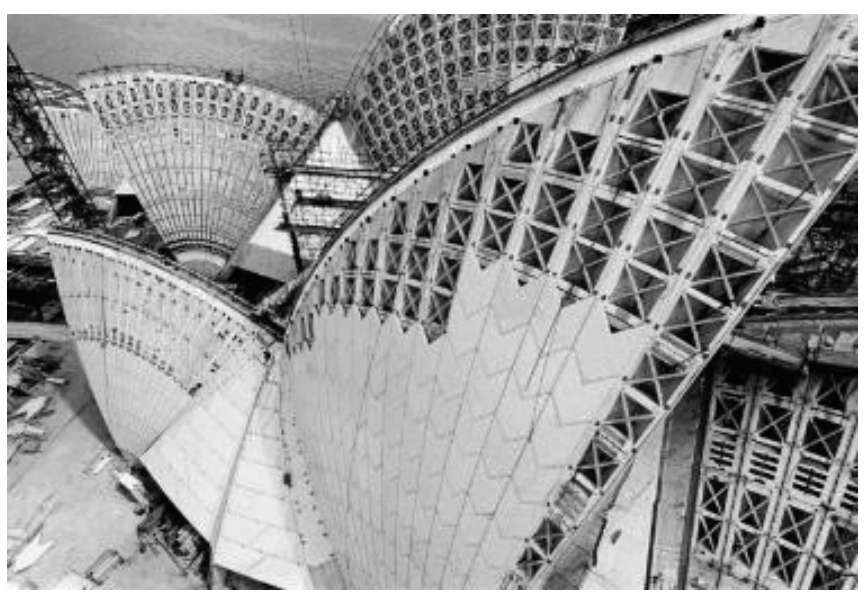

Figure 1.3: Cobertura da Opera House em Sydney Source:https:// duranvirginia.wordpress.com/2014/03/13/10-photographers-who-helped-make-architects-famous/

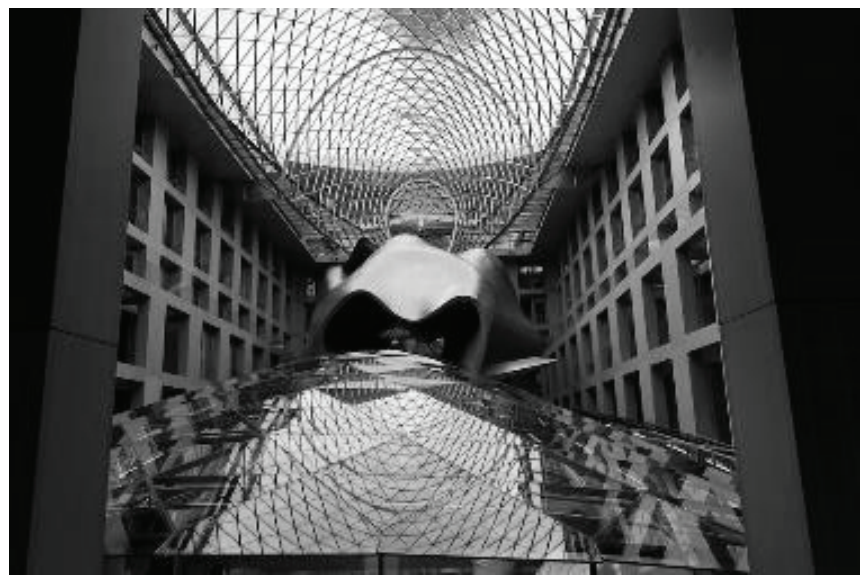

Figure 1.4: Cobertura do Pátio de em Parizer Platz, Berlin, Alemanha. Source: http://blaineharrington.photoshelter.com/image/ I0000eWUAdKHskMg 

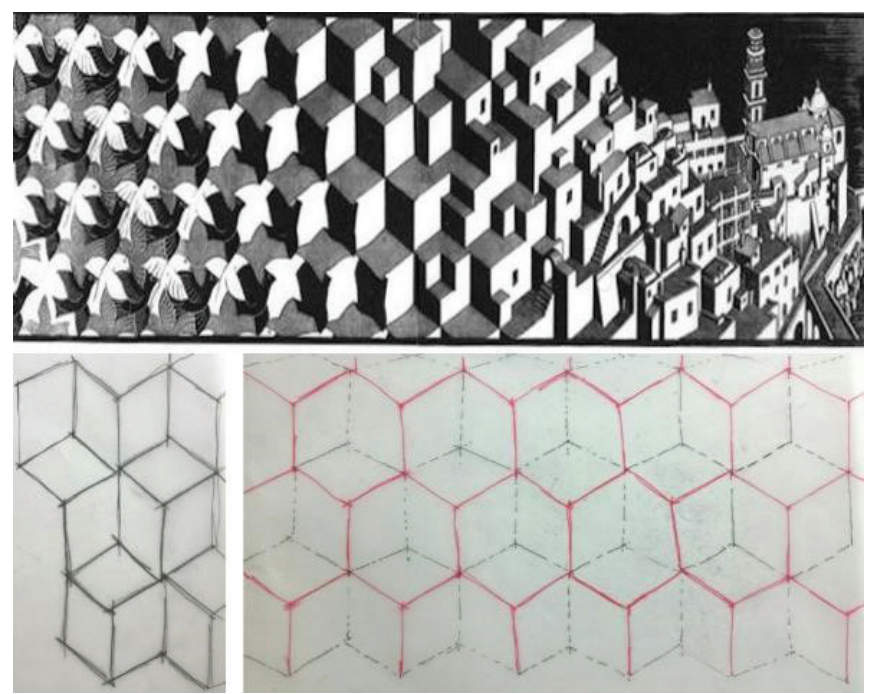

Figure 1.5: Inspiração da malha hexagonal da obra do M.C.Escher. Source; autoria nossa.

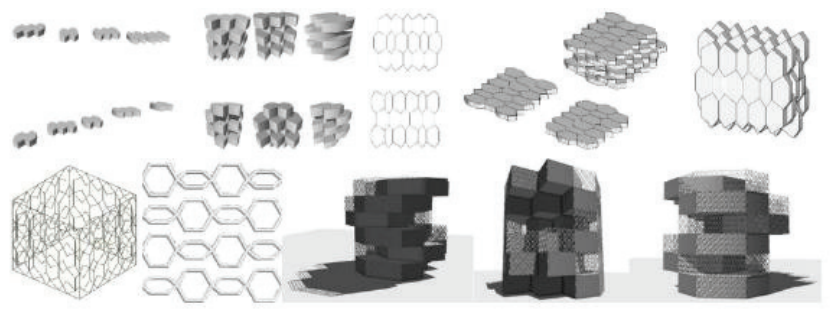

Figure 1.6: Simulações volumétricas a partir da malha Source; autoria nossa.

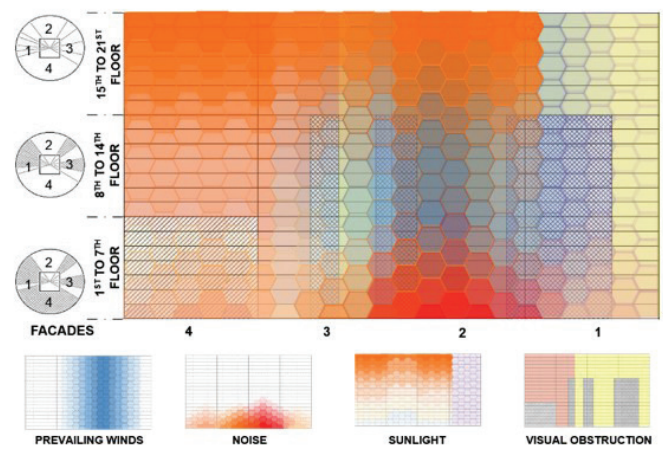

PERMEABILIDADE VISUAL

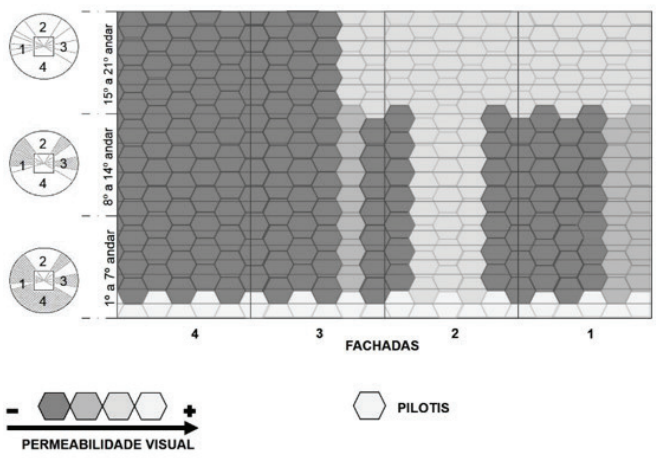

Figure 1.8: Mapeamento das Fachadas do Edifício Mostrando a Sobreposição das Análises de cada Condicionante Ambiental do Terreno; autoria nossa LUZ E SOMBRA - RESUMO DA INCIDÊNCIA SOLAR DAS FACHADAS - 8hrs, 10hrs,
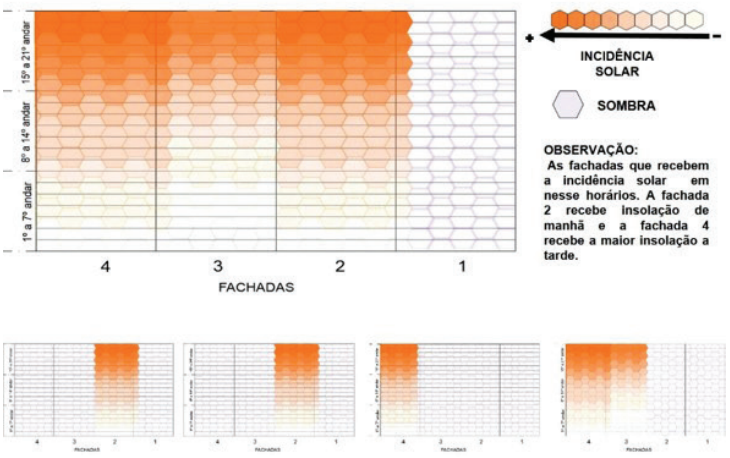

INCIDÊNCIA DE VENTOS PREDOMINANTES NAS FACHADAS
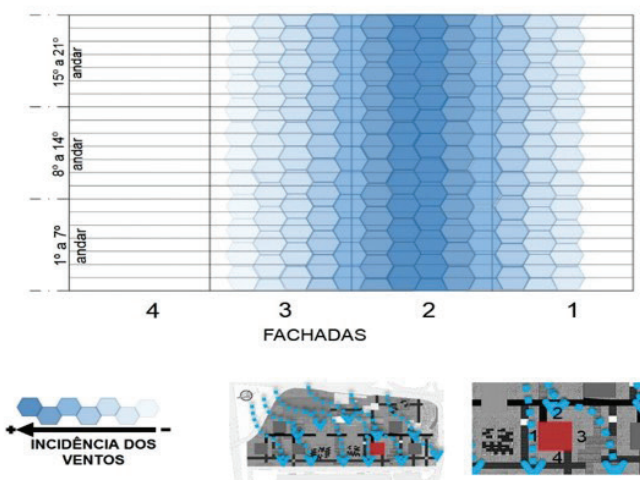

RUIDOS NAS FACHADAS DO EDIFICIO
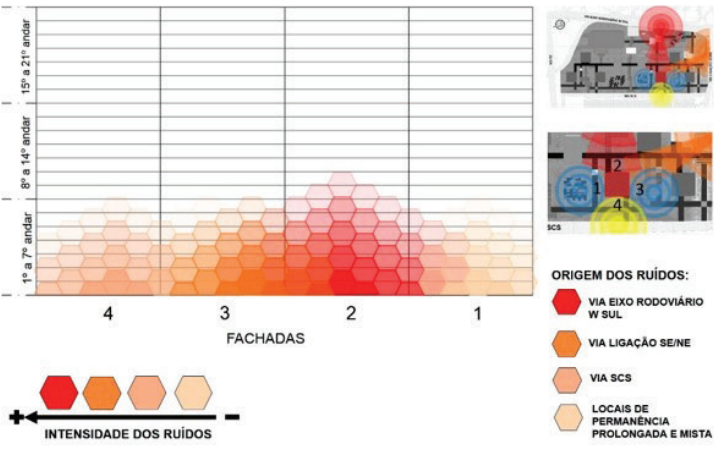

OBSTRUÇÃO VISUAL DEVIDO A PRESENÇA DOS EDIFÍCIOS VIZINHOS

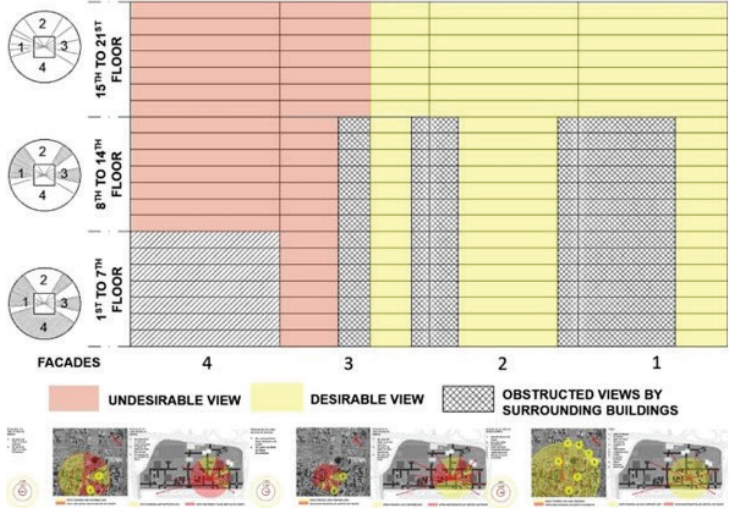

Figure 1.7: Análise dos Condicionantes Ambientais terreno escolhido no Setor Comercial Sul em Brasília. 
Analisamos as condicionantes do terreno escolhido no Setor Comercial Sul em Brasília, inserido portanto no contexto urbano, tais como ventos predominantes que vem de leste para oeste, pontos que tem ruídos significantes, vistas consideravelmente desejáveis, indesejáveis e até aquelas obstruídas pelos edifícios do entorno, além da insolação que as fachadas recebem do sol.

Apresentamos a seguir as imagens de todas as fachadas do edifício, mostrando o impacto de cada uma das variáveis, por grupos de andares, nas quais é possível visualizar a intensidade de cada uma delas de acordo com a gradação de cores. Na Figura 1.7 a seguir, por exemplo, apresentamos especificamente a incidência solar das 8 às 16 horas:

A seguir fizemos a sobreposição gráfica das análises de cada condicionante referente ao edifício estudado. Nosso objetivo foi identificar os problemas ambientais e vistas desejáveis e propor recomendações para a planta baixa de cada andar do edifício proposto (Figura 1.8).

Ressaltamos que na Figura 1.9 a seguir utilizamos duas malhas hexagonais de Tesselação para definir as formas básicas do projeto, no que se refere a composição da forma de cada andar do edifício e a compatibilização das malhas para a elaboração da estrutura. Assim as condicionantes ambientais estão influenciando diretamente a forma de cada andar e por conseguinte a estrutura do edifício.
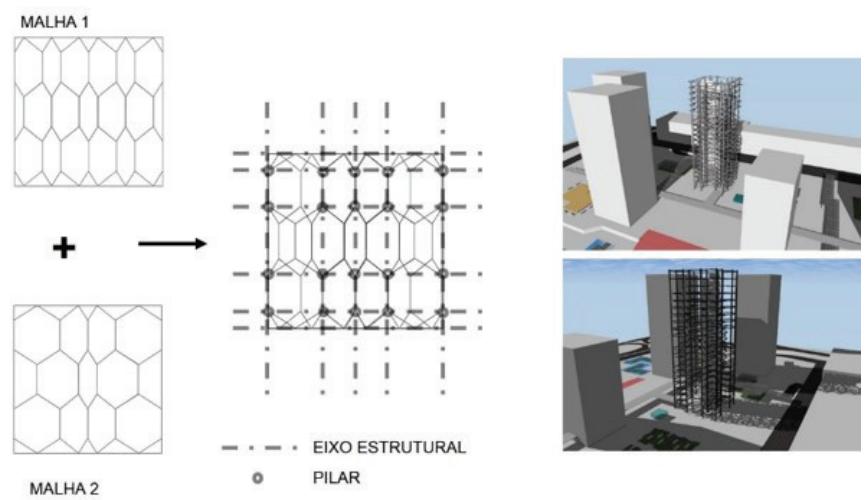

Figure 1.9: Simulações volumétricas a partir da malha Source; autoria nossa.

\section{Resultados}

O resultado deste mapeamento mostrou que existem regiões das fachadas doedifício que recebem simultaneamente pelo menos duas das condicionantes ambientais, em cada andar, com mais intensidade do que as outras.

Por exemplo, a fachada nordeste recebe muito vento e ruídosem pontosespecíficos. Por outrolado, a fachada sudoeste recebe alta intensidade de insolação. Em relação às outras condicionantes tais como ventos, ruídos, vistas obstruídas e permeabilidade visual a intensidade é considerada média ou baixa. Neste sentido veja a Figura 2.0 que mostra a intensidade destas condicionantes andar por andar.

Utilizamos um software de modelagem tridimensional, FormZ 7.3.4, para projetar e analisar as formas volumétricas no sentido de verificar se elas atendiam às referidas condicionantes ambientais estudadas.

Elaboramos uma escala mostrando desde a intensidade máxima até a mínima para analisar cada condicionante: exposição a luz solar, ventilação, ruído, permeabilidade visual até as vistas desejáveis de cada andar (Figura 2.2).

Nesta pesquisa elaboramos diversos diagramas para analisar e mapear cada condicionante isolada e conjuntamente de cada andar e propor uma solução específica para cada um deles. Portanto, em resposta a cada variável estudada propusemos uma planta baixa diferente para cada andar, por meio de adição ou subtração de certa quantidade de células hexagonais das malhas, conforme mostra Figura 2.1.

Propusemos que soluções arquitetónicas poderiam responder da seguinte maneira: onde houver muita insolação, ruídos e vento retira-se um ou mais células hexagonais, que vem de acordo com a malha de Tesselação hexagonal. Desta forma foi possível criar recuos em alguns andares e áreas que permitem aos usuários usufruí-las sem quaisquer desconfortos.

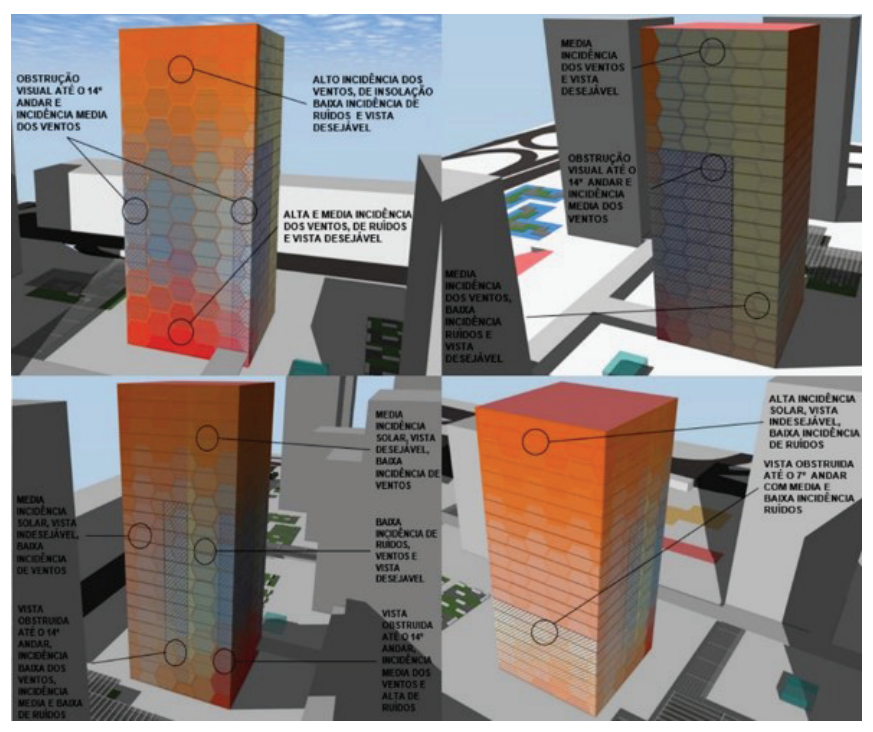

Figure 2.0: Vista de mapeamento das condicionantes do edifício projetado no Setor Comercial Sul, Asa Sul, Brasília, Distrito Federal. 
PROGRAMA

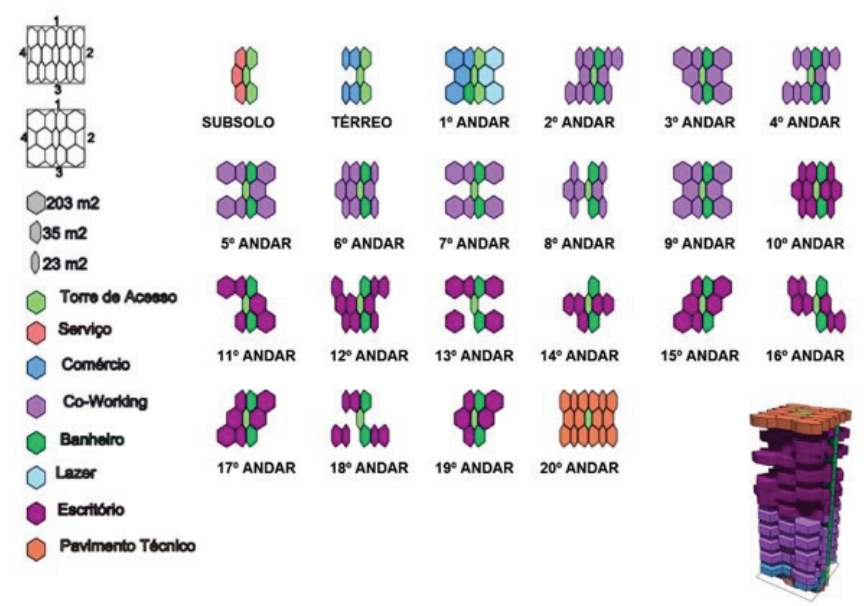

Figure 2.1: Programa de Necessidades do Edifício com cada andar com planta diferente devido a análise dos condicionantes ambientais.

SOLUÇÕES PARA CADA SITUAÇÃO

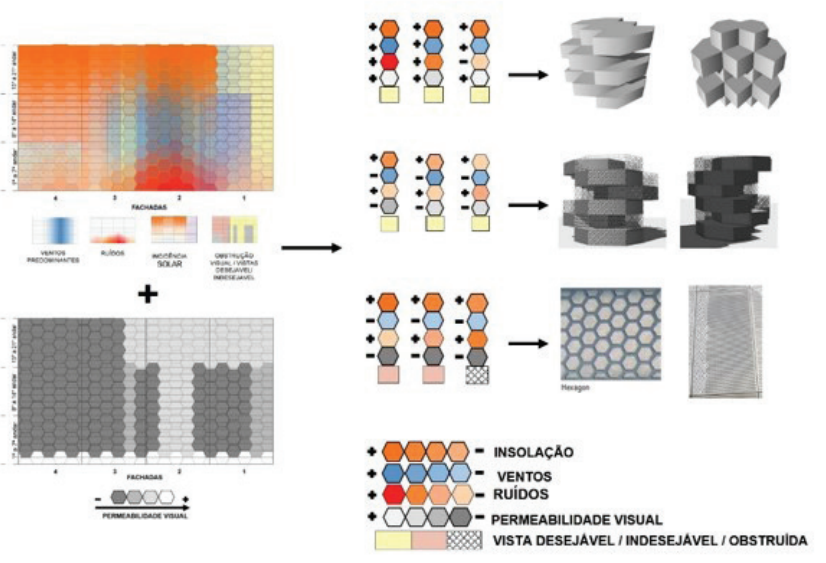

Figure 2.2: Solução Arquitetónicas para os Condicionantes Ambientais. Source; autoria nossa.

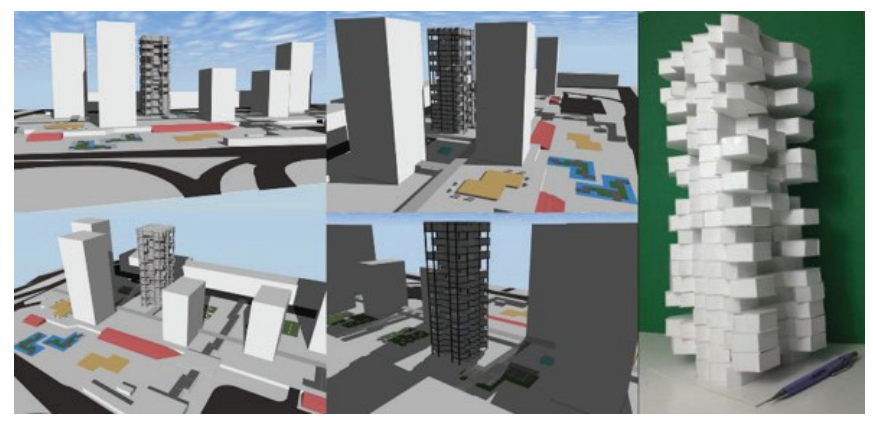

Figure 2.3: Modelo 3D e Maquete Física.Source; autoria nossa.

Em certos andares mantivemos os volumes hexagonais ou até acrescentamos alguns onde há vista desejável para se aproveitar. $\mathrm{O}$ resultado disto foi que cada andar teve uma planta baixa diferente (Figura 2.1), respondendo às condicionantes ambientais específicas da edificação.

Considerando os resultados encontrados também pesquisamos uma série de materiais que oferecem mais conforto para o edifício no sentido de reduzir insolação, ruídos e melhorar a ventilação. Nos casos em que a necessidade de um material ou elemento que controlasse melhor as condicionantes de insolação, ruídos e vistas consideradas obstruídas ou indesejáveis, pesquisamos materiais como um painel metálico perfurado utilizado em projetos arquitetónicos, da empresa Bausher, no sentido de permitir a luz e o vento passar e bloquear a vista indesejável.

Outra opção seria o uso de uma cortina de Hunter Douglas, do tipo "Alustra ${ }^{\circledR}$ Duette $₫$ Architella ${ }^{\circledR}$ ", que permite um controle maior de ruídos e de insolação ao mesmo tempo em que preserva e aproveita a vista desejável e interessante dos ambientes projetados de escritório, sala de "co-working", lazer e comercio.

Consideramos também nesta pesquisa a combinação de vidros temperados, do tipo "Low E", que permitem reduzir a insolação juntamente com a cortina de Hunter Douglas em ambientes como escritórios e salas, do tipo "co-working". Desta forma estes materiais combinados contribuem para resolver o problema de insolação e ruídos e ainda usufruir de vista desejável.

\section{Conclusões}

Demonstrando que a nossa hipótese de trabalho é promissora, os resultados finais obtidos desta pesquisa evidenciam que é necessário e factível projetar uma solução diferenciada para cada andar de um edifício novo em altura inserido no contexto urbano existente.

Ressaltamos ainda que a maior contribuição nova ao conhecimento apresentada nesta pesquisa consistiu na elaboração de um novo método de projetação utilizando a análise de diagramas individuais e conjunta das condicionantes existentes no contexto da edificação.

Destacamos ainda a importância e relevância da concepção de projeto a partir do conceito de Tesselação e da análise das condicionantes por meio de diagramas e de aplicação dos resultados obtidos na definição da forma da edificação no sentido produzir conforto ambiental.

Poder-se-ia argumentar que o entorno de uma edificação em altura é mutável ao longo do tempo e que a nossa proposta seria de aplicação limitada. Reconhecemos que, de fato, o entorno está continuamente mudando. Mas isto não ocorre de maneira nem mais rápida nem mais lenta do que as próprias alterações pós-ocupacionais internas do edifício. Portanto, qualquer conjunto de problemas ou condicionantes de projeto é mutável. Porém, o arquiteto sempre oferece e precisa oferecer uma resposta em referência a um momento histórico específico. Até que ponto sua proposta será uma resposta duradoura no caso do entorno, depende de fatores externos fora de seu controle.

As variáveis levadas em consideração neste trabalho não 
são passíveis de alteração rápida. O contexto urbano edificado de Brasília, assim como o de muitos centros urbanos tombados, é significativamente estável em termos de normas de gabarito e tipologias.

Por fim, a solução definitiva para a questão da mutabilidade do entorno seria encontrada em pesquisas sobre projeto paramétrico e arquiteturas responsivas, o que no momento se encontrava além dos limites do escopo deste trabalho.

\section{Referências}

IWAMOTO, LISA. (2009). Digital Fabrications - Architecture and Material Techniques. Princeton Architectural Press,
MOUSSAVI, FARSHID. (2009). The Function of Form. Harvard University,

KOLAREVIC, BRANKO. (2003). Architecture in the Digital Age - Design and Manufacturing.

KOLAREVIC, BRANKO. (2013). Manufacturing Material Effects: Rethinking Design and Making in Architecture.

LEE, Franklin. BEAURECUEIL, S. Anne. (2009). Articulated grounds: Mediating Environment and Culture. AA Agendas No.7. Architecture Association London.

KRAUEL, Jacobo. (2010). Contemporary Digital Architecture: Design and Techniques.

DELPINO, ROSSANA. (2014). Resumo de Curso. UniCEUB. 